\title{
Administrative versus clinical databases
}

\author{
Melanie P. Subramanian, MD, MPHS, ${ }^{\mathrm{a}}$ Yinin Hu, MD,${ }^{\mathrm{b}}$ Varun Puri, MD, MSCI, ${ }^{\mathrm{a}}$ and \\ Benjamin D. Kozower, MD, MPH ${ }^{\mathrm{a}}$
}

\begin{abstract}
Surgeons are under increasing pressure to measure surgical outcomes as a means of evaluating surgical quality and providing grounds for evidenced-based care. Clinicians and researchers have used observational research to address important questions pertaining to surgical outcomes; however, improper research practices are pervasive within observational research. Many studies use data mining of large databases instead of performing hypothesis-driven research with carefully selected data sources. ${ }^{1}$ The field of health services and outcomes research, especially within the cardiothoracic surgical literature, has seen an exponential growth in the number of observational studies that use data derived from administrative claims databases and large clinical registries. ${ }^{2}$ There are specific strengths and limitations associated with each of these data sources, and it is imperative that researchers, clinicians, and consumers of such studies understand the fundamental aspects of their selected database.
\end{abstract}

\section{ADMINISTRATIVE DATA}

Before choosing a suitable database, clinicians and researchers must understand key differences between data from administrative registries and data from clinical registries. These include differences in conception and design, geographic representation, definition of data variables, measurement of outcomes, and verification of data accuracy. ${ }^{2}$ Broadly speaking, administrative data are neither assembled nor designed for clinical purposes; rather, they are derived from claims data submitted to public or private groups for the purpose of reimbursement. ${ }^{3}$ Administrative databases obtain patient comorbidity and treatment information from diagnoses or procedure codes commonly using the International Classification of Diseases (ICD) and/or Current Procedural Terminology (CPT) coding systems. Thus, information on patient comorbidities, primary and secondary diagnoses, procedures, and complications are dependent on the thoroughness of the coding performed. Because administrative data are obtained from claims, there

\footnotetext{
From the a Division of Cardiothoracic Surgery, Department of Surgery, Washington University School of Medicine, St Louis, Mo and ${ }^{\mathrm{b}}$ Department of Surgery, Memorial Sloan-Kettering Cancer Center, New York, NY.

Received for publication Feb 7, 2020; revisions received March 22, 2020; accepted for publication March 26, 2020; available ahead of print June 24, 2020.

Address for reprints: Benjamin D. Kozower, MD, MPH, 660 S Euclid Ave, Campus Box 8234, St Louis, MO 63110 (E-mail: kozower@wustl.edu).

J Thorac Cardiovasc Surg 2021;162:1173-6

$0022-5223 / \$ 36.00$

Copyright (C) 2020 by The American Association for Thoracic Surgery

https://doi.org/10.1016/j.jtcvs.2020.03.183
}

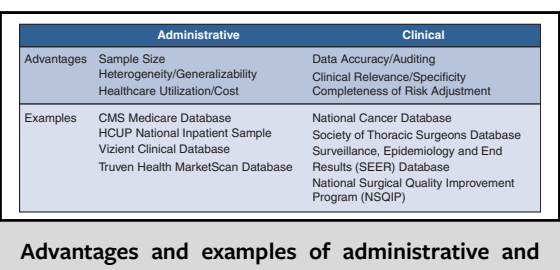
clinical databases.

CENTRAL MESSAGE

Investigators must understand the key differences between administrative and clinical data before selecting a suitable database for observational research.

This Invited Expert Opinion provides a perspective on the following papers: J Am Coll Surg. 2009; 209(5):551-556. 2009.08.008; J Am Coll Surg. 2016;223(4):557-558 https://doi.org/10.1016/j.jamcollsurg.2016.07.001; J Am Coll Surg. 2016;223(4):551-557.e4. https://doi.org/ 10.1016/j.jamcollsurg.2016.06.393.

See Commentary on page 1177.

is the unfortunate possibility of coding-related bias. Hospitals prioritize administrative coding to optimize reimbursement, not to enhance clinical accuracy. ${ }^{4}$ There is an incentive to provide codes related to diagnosis-related groups (DRGs) that are associated with greater reimbursement rather than with what are clinically accurate. In addition, undercoding/excluding certain diagnoses and/or procedures that are nonessential for billing is possible. For example, submissions of major surgical procedures may be accurate, but submissions of lower paymentgenerating diagnostic procedures or tests might not be as thorough. The accuracy of data input can be highly variable across hospitals based on the level of effort given to high-quality data entry and auditing, leading to issues with missing data in administrative data. In addition, conversions in coding systems (eg, ICD-9-CM to ICD-10$\mathrm{CM})$ can lead to potential errors in data entry and interpretation. ${ }^{2}$ Such practices can be detrimental when attempting to perform risk-adjusted analyses and can portray an inaccurate picture of a patient's treatment course.

Despite these limitations, administrative data are commonly used to address research questions, for several 
reasons. First, administrative datasets are relatively inexpensive and readily accessible. ${ }^{3}$ In addition, administrative data provide access to a large group of patients on a statewide, regional, or national level. The National Inpatient Sample (NIS) represents the largest national publicly available all-payer inpatient database. ${ }^{5}$ Developed by the Healthcare Cost and Utilization Project (HCUP) via funding from the Agency for Healthcare Research and Quality (AHRQ), the NIS contains inpatient hospital data from approximately $20 \%$ of short-term admissions to nonFederal HCUP-participating hospitals in the United States, regardless of payer. The NIS provides discharge weights that can be used to convert NIS sample data into nationallevel estimates. With discharge weights applied, the NIS provides accurate estimates for more than 35 million admissions. ${ }^{2,5}$ Administrative data files from the Centers for Medicare and Medicaid Services (CMS) represent another commonly used data source that provide data on $98 \%$ of adults at least 65 years of age enrolled in Medicare. ${ }^{6}$ This represents more than 45 million patients. These databases provide large, nationally representative sample sizes. Provided there is proper documentation of CPT codes, administrative data can used to measure the adoption and utilization of new technologies/techniques over time. Such data also can be useful for research questions aimed at estimating resource utilization, as they often contain charge data that can be converted to costs using published cost-to-charge ratios. ${ }^{3}$

The nature of the costs/charges must be taken into account. HCUP data reports charges reported by the hospital for inpatient services; however, other administrative databases report costs from the insurer perspective. CMS data report reimbursement from the insurer alone. Data from other administrative bases, such as the Truven Health MarketScan Database, report reimbursements from the insurer to the provider, as well as out-of-pocket costs paid by the patient.

In addition to resource utilization and cost data, administrative data can easily be used for longitudinal analyses. ${ }^{2,3}$ Administrative databases including the Truven Health MarketScan Database, CMS, and HCUP State Inpatient Database (SID) contain unique patient identifiers that can be linked to allow analyses for visits at different hospitals over time, allowing for longitudinal analyses.

\section{CLINICAL DATA}

Clinical databases are designed to collect information that is directly relevant to a particular medical specialty, disease process, or procedure. They are often used to assemble clinically relevant patient-level data that can be used for purposes of quality improvement and/or research. The scope of clinical databases can vary from a single institution to the national or even international level. Because clinical databases are designed with medical decision making in mind, they often include more accurate, detailed, and pertinent data on patient history, comorbidities, procedures, diagnostic testing, and outcomes. Large clinical databases are frequently run by medical associations or professional societies that have a vested interest in ensuring the collection and maintenance of high-quality data. These organizations deliver this standard through regular audits. This process requires an extraordinary amount of human and financial resources and buy-in from relevant stakeholders.

Within the cardiothoracic surgical literature are a number of clinical databases to select from for purposes of clinical research, quality improvement, and public reporting. Cancer registries, including the National Cancer Data Base (NCDB) and the Surveillance, Epidemiology, and End Results (SEER) database, have been used for countless studies in the general thoracic surgical literature. The NCDB, one of the largest hospital-based cancer registries, captures approximately $70 \%$ of incident cancers diagnosed annually in the United States. ${ }^{7}$ Created by a joint sponsorship of the American Cancer Society and the American College of Surgeons Commission on Cancer (CoC), the NCDB captures data submitted from more than $1500 \mathrm{CoC}$-accredited facilities. The NCDB ensures completeness and accuracy by mandating the prospective collection and submission of data from $\mathrm{CoC}$ sites and using specially trained data abstractors. ${ }^{7,8} \mathrm{Un}$ like administrative data, the NCDB contains detailed tumor characteristics (eg, TNM classification, clinical stage, pathological stage, histological subtype, grade), multimodality treatment (ie, chemotherapy, radiation, and/or surgery), 30and 90-day outcomes, and long-term overall survival. The accuracy and clinical relevance of the data make the NCDB superior to administrative data for several cancer-oriented studies; however, it is important to note that data might not be generalizable to patients who have received care at nonCoC-accredited facilities. ${ }^{2}$ In addition, not all outcomes are cancer-specific; for example, the NCDB cannot provide data on cancer recurrence and disease-free survival. ${ }^{7}$

Unlike the NCDB, the SEER database is a populationbased registry created by the National Cancer Institute. The database is currently a conglomeration of 21 state cancer registries selected to represent a diverse sample of the US population. ${ }^{9}$ Like the NCDB, the SEER has been touted for its data accuracy, which is ensured by routine auditing and trained data abstractors. In addition, SEER annually issues Data Quality Profiles which report metrics on missing data and reporting delays for each contributing registry. ${ }^{10}$ The SEER and NCDB database overlap on providing data variables on patient demographics and tumor characteristics; however, there are some important distinctions. SEER provides information on cancer-specific mortality but does not provide metrics of patient comorbidity burden, intent of therapy (curative vs palliative), or therapy beyond initial treatment. The SEER allows for linkage to other databases to partially overcome some of these limitations. ${ }^{11}$ The SEERMedicare database is one of the more commonly used linked 
databases. ${ }^{9}$ With the addition of administrative claims data, SEER data are augmented with information on comorbidities, treatment-related complications, and more concrete chemotherapy/radiation data (including type, duration, and strength/dosing). ${ }^{12}$ However, the SEER-Medicare linked database is much more complicated and requires high-level data management experience.

Outside of cancer-focused registries, the cardiothoracic surgical field is fortunate to have the robust Society of Thoracic Surgeons (STS) database and its 4 subcomponents: Adult Cardiac, Congenital Cardiac, Mechanical Circulatory Support, and General Thoracic Surgery. ${ }^{13,14}$ The STS database is one of the most comprehensive cardiothoracicfocused clinical registries available. With routine data collection on patient sociodemographic information, preoperative evaluation, functional status, specific comorbidities, diagnostic testing, relevant laboratory values, clinical and pathologic staging, operative details, and multiple postoperative major and minor complications, the STS database provides significant granularity with a high degree of data quality. ${ }^{13}$ Additional enhancements to the STS are being made to improve longitudinal data collection beyond 30-day outcomes. The STS will soon link with the Centers for Disease Control and Prevention's National Death Index (NDI) to provide information on time and cause of death. ${ }^{15,16}$ With annual audits to ensure a high degree of completeness and accuracy, the STS represents a robust database for health services research and quality improvement.

There has been an increasing emphasis on the use of large databases for public reporting of surgical outcomes at the hospital and surgeon levels. These are often based on riskadjusted models of postoperative mortality, complications, and/or readmissions. Such models are created to measure quality of care and help the public select hospitals and/or physicians. Creation of these models provides crucial lessons in the importance of understanding sources of data, especially when using claims data. Previous studies have highlighted the inadequacies of administrative data when used to measure surgical quality for common cardiothoracic procedures.

Prasad and colleagues ${ }^{17}$ performed a retrospective study attempting to identify differences in administrative and clinical databases in a cohort of patients who underwent cardiac surgery. The administrative data source was the University HealthSystem Consortium (UHC), which is geared toward providing risk-adjusted models for purposes of quality assessment and quality improvement. The UHC (now Vizient, Inc) collects patient information from discharge abstracts from an alliance of 101 academic centers and their affiliate hospitals. ${ }^{18}$ It performs risk adjustment based on patient severity determined by the All Patient Refined Diagnosis-Related Groups. Prasad and colleagues ${ }^{17}$ compared the accuracy of data maintained by the UHC database against the STS Cardiac Surgery Database. ${ }^{17}$ They identified a cohort of 2927 patients who underwent coronary artery bypass grafting (CABG), aortic valve replacement (AVR), and mitral valve replacement (MVR) and compared data accuracy for demographic information, comorbidities, mortality rates, and postoperative complications. Both databases demonstrated similarity in all patient data, with the exception of postoperative complications. UHC data did not have sufficient granularity to include common postoperative complications, such as atrial fibrillation. The UHC recorded stroke, postoperative shock, and perioperative hemorrhage as the top 3 postoperative complications. This differed from STS data, which identified atrial fibrillation, prolonged ventilation, and reoperation for bleeding/tamponade as the leading postoperative complications. The STS-listed complications seem much more likely for a cardiac surgical cohort. Prasad and colleagues highlighted how the use of clinical databases allows for a more precise study of cardiac surgical patients and more accurately reflects clinical reality.

Kozower and colleagues ${ }^{19}$ further demonstrated the potential pitfalls of using administrative data for quality assessment. They compared postoperative mortality risk prediction models derived from the UHC and STS databases. Specifically, they compared the predictive performance and calibration measures for the 2 models using an institutional cohort of $2171 \mathrm{pa}-$ tients undergoing CABG, AVR, MVR, or combined AVR/ CABG or MVR/CABG. The authors noted that that when using the entire study cohort, the UHC model had a higher predicted performance compared with the STS model; however, they identified important methodologic differences between the risk models of STS and UHC. Traditionally, prediction models that are created to evaluate a postoperative outcome should include only preoperative variables; however, UHC models included postoperative complications as predictors in their risk model, which led to a false estimation of risk and inflated the predictive capacity of the UHC. When the authors applied both models in a cohort of patients without any postoperative complications, the performance of the UHC model declined significantly (C statistic of 0.88 to 0.49 ), whereas the STS model was relatively unaffected. The authors concluded that given these methodological flaws, the UHC models should not be used for quality benchmarking.

Whereas these previous studies highlighted limitations associated with administrative databases, other studies have demonstrated poor generalizability of high-quality clinical databases. LaPar and colleague ${ }^{20}$ highlighted differences in the reporting of outcomes after esophagectomy for cancer using commonly used clinical and administrative databases. They compared esophageal cancer resection mortality, hospital length of stay, and volume from 2002 to 2008 between the STS General Thoracic Surgery Database (GTSD), the National Surgical Quality Improvement Project (NSQIP), and the NIS. The authors noted that mortality was significantly different among databases, with the clinical databases reporting lower mortality rates (NIS, 
$6.1 \%$; NSQIP, $2.6 \%$; GTSD, $3.2 \%$ ). In addition, the median length of stay was shorter in the GTSD (NIS, 12 days; NSQIP, 12 days; GTSD, 10 days). The GTSD contained a higher proportion of tertiary care facilities and cardiothoracic or general thoracic specialists compared with general surgeons. Compared with the NIS, the GTSD reported only a fraction of the esophagectomies performed-only $36 \%$ of estimated surgeries performed annually in the United States. Although the number of hospitals contributing to the GTSD continues to rise every year, the authors concluded that further increases in participation are needed.

These studies exemplify the importance of performing a critical evaluation of the databases used in observational research, especially when it comes to limitations and potential sources of bias. In addition, study investigators must provide clear and transparent reporting on their use of databasederived research. In addition to the Strengthening the Reporting of Observational Studies in Epidemiology (STROBE) guidelines, additional recommendations have been developed specifically for investigations that use either administrative or clinical data. ${ }^{21}$ The Reporting of Studies Conducted Using Observational Routinely Collected Health Data (RECORD) statement was created as an extension of STROBE guidelines to improve reporting practices in observational studies that use routinely collected health information. $^{22,23}$ The RECORD statement advises investigators to include explicit details on the type of database used, codes and algorithms for study population selection, data cleaning techniques, data linkage practices, and assessments of data quality. ${ }^{23}$ In addition, the guidelines recommend that investigators be transparent regarding potential limitations of the database that could lead to misclassification bias, unmeasured confounding, missing data, and changing eligibility over time. By considering these steps, investigators will not only improve the integrity of reporting, but will also be compelled to reassess the suitability of a selected database for each new research question.

Clinicians and researchers have a variety of databases to choose from when performing observational research. Carefully considering the selection of a database is almost as important as carrying out the study itself. Investigators have a responsibility to understand the advantages and limitations of their selected database. A comprehensive understanding of the differences between administrative and clinical datasets is an important first step.

\section{Conflict of Interest Statement}

The authors reported no conflicts of interest.

The Journal policy requires editors and reviewers to disclose conflicts of interest and to decline handling or reviewing manuscripts for which they may have a conflict of interest. The editors and reviewers of this article have no conflicts of interest.

\section{References}

1. Lee CH, Yoon HJ. Medical big data: promise and challenges. Kidney Res Clin Pract. 2017:36:3-11.

2. Groth SS, Habermann EB, Massarweh NN. United States administrative databases and cancer registries for thoracic surgery health services research. Ann Thorac Surg. 2020;109:636-44.

3. Hu Y, Puri V, Kozower BD. Rationale for and use of large databases. In: Shields TW, LoCicero J III, Reed CE, Feins RH, eds. Shields' General Thoracic Surgery. 7th ed. Philadelphia: Lippincott Williams \& Wilkins; 2009.

4. van Walraven C, Austin P. Administrative database research has unique characteristics that can risk biased results. J Clin Epidemiol. 2012;65:126-31.

5. Healthcare Cost and Utilization Project (HCUP). Overview of the national (nationwide) inpatient sample (NIS); 2019. Available at: https://www.hcup-us. ahrq.gov/nisoverview.jsp. Accessed May 25, 2020.

6. Hashimoto RE, Brodt ED, Skelly AC, Dettori JR. Administrative database studies: goldmine or goose chase? Evid Based Spine Care J. 2014;5:74-6.

7. Stewart AK, Bland KI, McGinnis LS Jr, Morrow M, Eyre HJ. Clinical highlights from the national cancer data base, 2000. CA Cancer J Clin. 2000; 50:171-83.

8. American College of Surgeons, National Cancer Database, CoC quality of care measures. CoC measures for quality of cancer care. Available at: https://www. facs.org/quality-programs/cancer/ncdb/qualitymeasurescocweb. Accessed May 25, 2020.

9. National Cancer Institute. Surveillance, Epidemiology, and End Results Program. About the SEER registries; 2019. Available at: https://seer.cancer. gov/registries/. Accessed May 25, 2020.

10. Hankey BF, Ries LA, Edwards BK. The surveillance, epidemiology, and end results program: a national resource. Cancer Epidemiol Biomarkers Prev. 1999;8:1117-21.

11. Park HS, Lloyd S, Decker RH, Wilson LD, Yu JB. Limitations and biases of the surveillance, epidemiology, and end results database. Curr Probl Cancer. 2012; 36:216-24.

12. Warren JL, Klabunde CN, Schrag D, Bach PB, Riley GF. Overview of the SEER-Medicare data: content, research applications, and generalizability to the United States elderly population. Med Care. 2002;40(8 Suppl):IV3-18.

13. Jacobs JP, Shahian DM, D’Agostino RS, Mayer JE Jr, Kozower BD, Badhwar V, et al. The Society of Thoracic Surgeons national database 2018 annual report. Ann Thorac Surg. 2018;106:1603-11.

14. The Society of Thoracic Surgeons. STS national database; 2019. Available at: https://www.sts.org/registries-research-center/sts-national-database. Accessed May 25, 2020.

15. Fernandez FG, Kosinski AS, Tong BC, Furnary AP, Feng L, Onaitis M, et al. Lack of correlation between short- and long-term performance after lung cancer surgery. J Thorac Cardiovasc Surg. 2019;157:1633-43.e3.

16. The Society of Thoracic Surgeons. 'Game-changing' data to supplement STS National Database. STS News, Fall 2019. Available at: https://www.sts.org/ publications/sts-news/game-changing-data-supplement-sts-national-database. Accessed May 25, 2020.

17. Prasad A, Helder MR, Brown DA, Schaff HV. Understanding differences in administrative and audited patient data in cardiac surgery: comparison of the University HealthSystem Consortium and Society of Thoracic Surgeons databases. J Am Coll Surg. 2016;223:551-7.e4.

18. Vizient. Vizient member networks; 2019. Available at: https://www.vizientinc com/our-networks/vizient-member-networks. Accessed May 25, 2020.

19. Kozower BD, Ailawadi G, Jones DR, Pates RD, Lau CL, Kron IL, et al. Predicted risk of mortality models: surgeons need to understand limitations of the University HealthSystem Consortium models. J Am Coll Surg. 2009;209:551-6.

20. Lapar DJ, Stukenborg GJ, Lau CL, Jones DR, Kozower BD. Differences in reported esophageal cancer resection outcomes between national clinical and administrative databases. J Thorac Cardiovasc Surg. 2012;144:1152-7.

21. Vandenbroucke JP, von Elm E, Altman DG, Gøtzsche PC, Mulrow CD, Pocock SJ, et al. Strengthening the reporting of observational studies in epidemiology (STROBE): explanation and elaboration. Int J Surg. 2014;12: 1500-24.

22. Langan SM, Benchimol EI, Guttmann A, Moher D, Petersen I, Smeeth L, et al. Setting the RECORD straight: developing a guideline for the REporting of studies Conducted using Observational Routinely collected Data. Clin Epidemiol. 2013;5:29-31.

23. Benchimol EI, Smeeth L, Guttmann A, Harron K, Moher D, Petersen I, et al. The REporting of studies Conducted using Observational Routinely-collected health Data (RECORD) statement. PLoS Med. 2015;12:e1001885. 